\title{
Voltage-Induced Sample Release from Anion Exchange Supports in Capillary Electrochromatography
}

\author{
Shinya Kitagawa and Takao Tsuda ${ }^{\dagger}$ \\ Department of Applied Chemistry, Nagoya Institute of Technology, Gokiso, Showa, Nagoya 466-8555, Japan
}

\begin{abstract}
An electric field application on ion exchange packing supports induced the release of sample solutes from the supports. When the electric field was applied temporary on the column, this phenomenon was observed as an increase in the pulsed concentration in the mobile phase. Namely a peak emerges on a chromatogram. The effect of the applied voltage on the ion exchange resin was examined by using a tiny mini-column. The sample solution was continuously passed through and the variations of effluent composition with and without application of electric field were analyzed. It was observed that amounts of increments of molar concentration of both benzoic acid and benzenesulfonic acid in mobile phase depended on the strength of the electric field. A theory of variation of distribution constant due to the electric field is proposed.
\end{abstract}

Keywords Electric field, pulse, distribution coefficient alternation, anion exchange, electrochromatography

Capillary electrochromatography ${ }^{1-7}$ is one of the topics in the separation sciences as it plays a crucial role in the stages developing nowadays. In this method, an electric field is applied along a capillary column, and the electric field introduces two new factors, electroosmotic flow and electrophoretic migration of solutes. These two factors do not exist in ordinary liquid chromatography. These two new factors influence the behavior of solutes in the mobile phase. Namely, both factors contribute to the apparent flow velocities of solutes.

During an experiment ${ }^{7,8}$ of electrochromatography using ion exchange supports, an interesting phenomenon was observed. When an electric field was applied on the column, the baseline of the chromatogram fluctuated, and after a while it assumed its original position. ${ }^{8,9}$ Namely, the applied voltage causes a temporary increase in the solute concentration indicated by a peak without any sample injection. Here, the electric field probably induces the variation of the distribution coefficient. Therefore, the electric field has an effect not only on separations due to the electrophoretic mobility in mobile phase, but also on the equilibrium between solutes and packing supports. It is necessary to understand this phenomenon for the further development of electrochromatography. And this phenomenon gives new information about the interaction between solute and ion exchange supports.

In the present report, the chromatographic behaviors of a mixture of two compounds were examined by using a tiny mini-column (we called this mini-column as mini-bed) under a continuous sample introduction with and without applied voltage. For detecting the

$\doteqdot$ To whom correspondence should be addressed. variation of the two compounds a multi-scanning detector was used. Based on this phenomenon, a theory is now being proposed.

\section{Theory}

Under the condition of equilibrium, an electrochemical potential for solute $\alpha$ in mobile phase, $\mu_{\alpha, \mathrm{m}}$, is equal to its electrochemical potential in stationary phase, $\mu_{\alpha, s}$, Namely,

$$
\mu_{\alpha, \mathrm{m}}=\mu_{\alpha, \mathrm{s}}
$$

Each electrochemical potential is described as follows,

$$
\begin{aligned}
& \mu_{\alpha, \mathrm{m}}=\mu_{\alpha, \mathrm{m}}^{\circ}+R T \ln \left[\alpha_{\mathrm{m}}\right]+z F \phi_{\mathrm{m}} \\
& \mu_{\alpha, s}=\mu_{\alpha, \mathrm{s}}^{\circ}+R T \ln \left[\alpha_{\mathrm{s}}\right]+z F \phi_{\mathrm{s}}
\end{aligned}
$$

where $\mu^{\circ}, R, T,[\alpha], z, F$ and $\phi$ are standard chemical potential, gas constant, temperature, concentration of solute $\alpha$, number of electric charge, Faraday constant and internal potential, respectively. Subscripts $\mathrm{m}$ and $\mathrm{s}$ mean mobile and stationary phases, respectively. Combining Eqs. (1), (2) and (3) gives

$$
\ln \left(\left[\alpha_{\mathrm{s}}\right] /\left[\alpha_{\mathrm{m}}\right]\right)=-\left(\Delta \mu_{\alpha}^{\circ}+z F \phi_{\mathrm{D}}\right) /(R T)
$$

where $\Delta \mu_{\alpha}^{\circ}=\mu_{\alpha, s}^{\circ}-\mu_{\alpha, \mathrm{m}}^{\circ}, \phi_{\mathrm{D}}=\phi_{\mathrm{s}}-\phi_{\mathrm{m}}$. The left side of Eq.(4) is equal to $\ln K$, where $K$ is the distribution coefficient.

Rudge $^{10}$ has assumed that the sample adsorption depends on the electric field. Therefore, it is reasonable to assume that the potential difference between mobile and stationary phases is changed by application 
of voltage along the mini-bed. It would be expressed as $\phi_{\mathrm{D}}+f V$, where $V$ and $f$ are an applied voltage along the mini-bed and a proportional constant for voltage variation in local potential, respectively. By using this assumption, Eq. (4) is rewritten to

$$
\ln K=-\left(\Delta \mu_{\alpha}^{\circ}+z F\left(\phi_{\mathrm{D}}+f V\right)\right) /(R T)
$$

and

$$
K(V)=K_{0} \exp (-\mathrm{z} F f V / R T)
$$

where $K(V)$ is the distribution coefficient at the application of $V$ voltage, and $K_{0}$ is the distribution constant at $V=0$. The $V$ in Eq. (6) is variable, so the distribution constant is able to vary.

By using plate theory, when the distribution coefficient is varied from $K$ to $K^{\prime}$, each total sample mass in plate $n, M_{n}$ and $M_{n}{ }^{\prime}$, respectively, can be expressed as follows,

$$
\begin{aligned}
& M_{n}=\left[C_{\mathrm{s}}\right] v_{\mathrm{s}}+\left[C_{\mathrm{m}}\right] v_{\mathrm{m}} \\
& M_{n}{ }^{\prime}=\left[C_{\mathrm{s}}{ }^{\prime}\right] v_{\mathrm{s}}+\left[\mathrm{C}_{\mathrm{m}}{ }^{\prime}\right] v_{\mathrm{m}}
\end{aligned}
$$

where $v_{\mathrm{s}}$ and $v_{\mathrm{m}}$ are local theoretical plate volumes of stationary and mobile phases, respectively. If the variation from $K$ to $K^{\prime}$ induced by applied voltage is short enough, the mass transfer between each plates would be zero. Therefore, $M_{n}$ in Eq. (7) and $M_{n}{ }^{\prime}$ in Eq. (8) are equal. Combination of Eqs. (7) and (8) leads to the alternative concentration in mobile phase. $\left[C_{\mathrm{m}}{ }^{\prime}\right]$ is expressed as follows.

$$
\left[C_{\mathrm{m}}{ }^{\prime}\right]=(K \beta+1) /\left(K^{\prime} \beta+1\right)\left[C_{\mathrm{m}}\right]
$$

where $\beta$ is the phase ratio, $\beta=v_{\mathrm{s}} / v_{\mathrm{m}}$.

Combination of Eqs. (6) and (9) provides

$$
\left[C_{\mathrm{m}}\right](V)=\left(K_{0} \beta+1\right) /\left(K_{0} \beta \exp (-z F f V / R T)+1\right)\left[C_{\mathrm{m}}\right]
$$

where $\left[C_{\mathrm{m}}\right](V)$ is the alternative concentration with application of $V$ voltage. The increment/decrement ratio of sample concentration is given as the following equation.

$$
\Delta C_{\mathrm{m}}(V) /\left[C_{\mathrm{m}}\right]=\left(K_{0} \beta+1\right) /\left(K_{0} \beta \exp (-z F f V / R T)+1\right)-1
$$

In Eq. (11), $K_{0} \beta$ can be expressed as $k_{0}^{\prime}$, which is the capacity factor at $V=0$. So, the Eq. (11) becomes

$$
\Delta C_{\mathrm{m}}(V) /\left[C_{\mathrm{m}}\right]=\left(k_{0}^{\prime}+1\right) /\left(k_{0}^{\prime} \exp (-z F f V / R T)+1\right)-1
$$

Equation (12) gives the relationship between applied voltage and alternation ratio of concentration. By using Eq. (12), the values of $k^{\prime}$ and $-z F f / R T$ are estimated from data of $V$ and $\Delta C_{\mathrm{m}}(V) /\left[C_{\mathrm{m}}\right]$ obtained by experiments.

\section{Experiment}

\section{Apparatus}

The schematic diagram of the apparatus used is shown in Fig. 1. The apparatus was composed of a pump (LC-10A, Shimadzu, Kyoto), a multi-scanning detector (SPD-M10A, Shimadzu), a voltage supply (PLD-36-1.2, Matsusada Precision, Shiga, Japan) and a home-made tiny column, called a mini-bed. The minibed was composed of a glass tubing (i.d. $0.7 \mathrm{~mm}$ ), two platinum tubings, fused silica capillaries (i.d. $0.2 \mathrm{~mm}$ ) and two Teflon filters (thickness $0.1 \mathrm{~mm}$ ). The minibed was packed with anion exchange resins (column length packed, $1 \mathrm{~mm}$; packing supports, TSKgel ICANION-SW and particle diameter $5 \mu \mathrm{m}$, Tosoh, Tokyo, Japan). All three tubings (glass, platinum and fused silica capillary tubings) were sealed together with epoxy glue. The platinum tubes were used as electrodes, and the platinum tube at inlet side was always grounded. Thus, an expression of "applied $+\alpha \mathrm{V}$ " means that $+\alpha \mathrm{V}$ was applied at the outlet platinum tube.

\section{Samples}

Three sample solutions: (a) $0.1 \mathrm{mM}$ benzenesulfonic acid sodium salt aqueous solution; (b) $0.1 \mathrm{mM}$ benzoic acid potassium salt aqueous solution; (c) the mixture of $0.1 \mathrm{mM}$ benzenesulfonic acid sodium salt and $0.01 \mathrm{mM}$ benzoic acid potassium salt aqueous solution were used. All of these reagents were guaranteed grade, and were supplied from Wako Pure Chemical Industries, Ltd., Kyoto.

\section{Chromatographic system}

A liquid chromatographic system was used for the

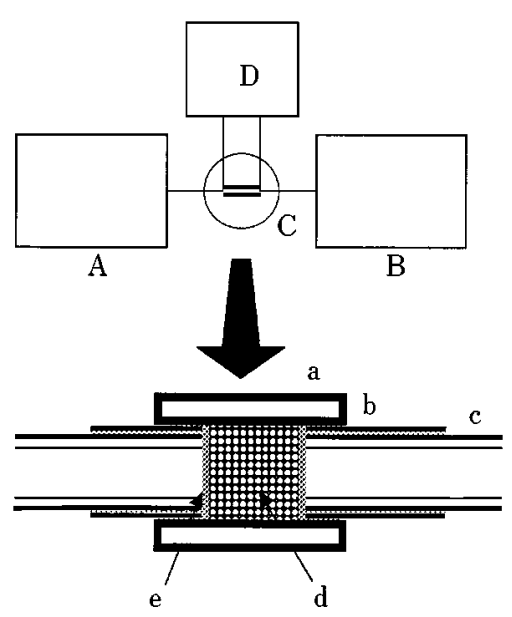

Fig. 1 A schematic diagram of the apparatus used for the experiment of concentration alternation induced by electric field. (A) pump; (B) multi scanning detector; (C) mini-bed; (D) dc power supply. A projected view of a mini-bed: (a) glass tube $(0.7 \mathrm{~mm}$ i.d. $)$; (b) platinum tubes used as electrodes; (c) fused-silica capillaries (200 $\mu \mathrm{m}$ inner diameter); (d) anion exchange packing supports; (e) Teflon filter. 
measurement of capacity factors of benzoic acid and benzenesulfonic acid. The chromatograph was composed of a pump (LC-6A, Shimadzu, Kyoto), a homemade capillary column $(0.2 \mathrm{~mm}$ inner diameter and 150 $\mathrm{mm}$ in length; packing supports: TSKgel IC-ANIONSW), an injector (Model 7125, Rheodyne, Co., Contati, USA), a UV detector (CE-970, JASCO, Tokyo) and a home-made split injection.

\section{Operational procedure}

Sample solution was made to flow through a minibed continuously by a pump, at flow rate of $0.1 \mathrm{ml} / \mathrm{min}$, during the experiment. After the equilibrium condition was obtained, the electric voltage, -5 to $-30 \mathrm{~V}$, was applied. The effluent coming out from the mini-bed was taken directly to the multi-scanning detector. The concentration variations in effluent with and without applied voltage were obtained by the analyses with the multi-scanning detector.

\section{Results and Discussion}

\section{Typical phenomenon induced by applied voltage}

The result of one of the typical phenomena induced by the application of voltage on the mini-bed is shown in Fig. 2. The mixture of $0.1 \mathrm{mM}$ benzenesulfonic acid sodium salt and $0.01 \mathrm{mM}$ benzoic acid potassium salt aqueous solution was used. The sample solution was continuously introduced into mini-bed by a pump, at a

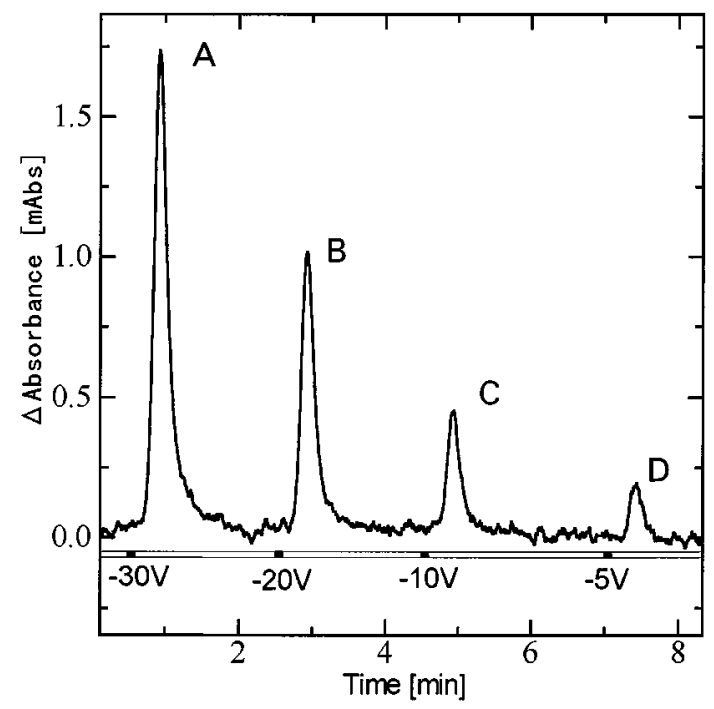

Fig. 2 Typical phenomenon induced by application of electric voltage. These peaks were obtained by an application of a pulsed electric voltage of $-30,-20,-10$ or $-5 \mathrm{~V}$. Black and white bars under the chromatogram mean periods with application of voltage and periods without applied voltage, respectively. All duration periods of applied voltage are $6 \mathrm{~s}$. Sample solution: the mixture of $0.1 \mathrm{mM}$ benzenesulfonic acid sodium salt aqueous solution and $0.01 \mathrm{mM}$ benzoic acid potassium salt aqueous solution; flow rate of sample solution: 0.1 $\mathrm{ml} / \mathrm{min}$. Detection: $254 \mathrm{~nm}$. flow rate of $0.1 \mathrm{ml} / \mathrm{min}$. Peaks of A, B, C and D in Fig. 2 were obtained by the pulsed applied voltage of $-30 \mathrm{~V}$, $-20 \mathrm{~V},-10 \mathrm{~V}$ and $-5 \mathrm{~V}$, respectively. Black and white bars under the chromatogram mean periods with application of voltage and periods without applied voltage, respectively, and all duration periods of applied voltage are $6 \mathrm{~s}$. These peaks were induced by the temporary alternation of concentration of UV absorbable solutes in the mobile phase. These peaks indicated the increments of the concentration of benzoic acid and benzenesulfonic acid. The concentration increment was derived by application of electric field, and was proportional to the value of applied voltage. By applying pulsed voltage, sample solute was released or detached from the surface of ion exchange supports.

When positive applied voltage was used, negative peak was obtained. Therefore these phenomena recognize the direction of electric field.

\section{Spectra of peaks induced by application of voltage}

Two sample solutions of (a) $0.1 \mathrm{mM}$ benzoic acid aqueous solution and (b) $0.1 \mathrm{mM}$ benzenesulfonic acid sodium salt aqueous solution gave the spectra in Fig. 3-A and -B, respectively. Both spectra of A and B were obtained at the maximum position of each peak, which was induced by six seconds pulse applications of -20 V. The vertical axis in Fig. 3 was the absorption increment in ultraviolet region. Benzoic acid gave a broad absorption band, while benzenesulfonic acid gave fine resolved absorption bands and showed three different absorption maxima called benzenoid absorption.

Each concentration increments induced by the applications of voltage was estimated by using the values of molecular absorption coefficients of benzenesulfonic

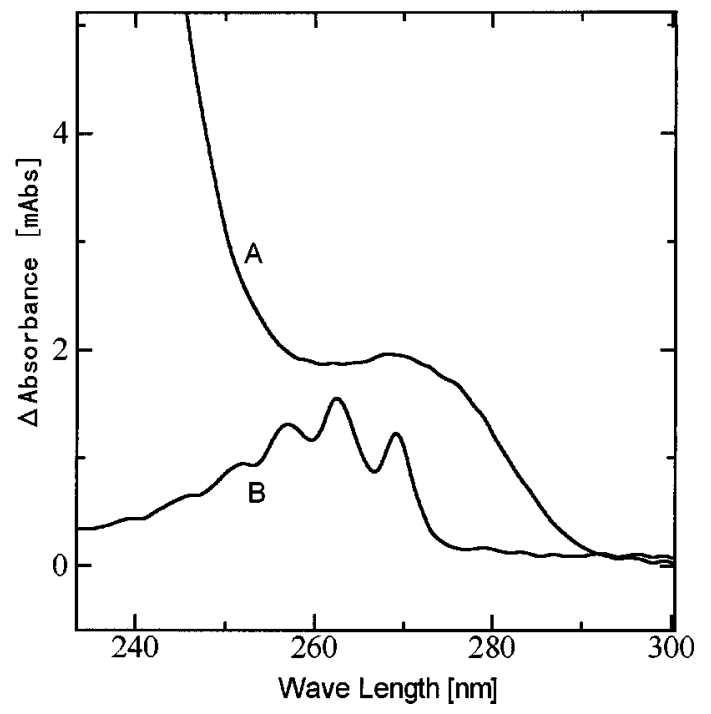

Fig. 3 Spectra of peaks induced by applied pulse voltage. Vertical line ( $\Delta$ absorbance) is the difference of the absorbent values between effluents with and without applied voltage. Sample solutions: $0.1 \mathrm{mM}$ benzoic acid potassium salt [A] and $0.1 \mathrm{mM}$ benzenesulfonic acid sodium salt [B]. 
acid and benzoic acid. ${ }^{11}$ The increments of concentration were calculated by using the following equation.

$$
\Delta \operatorname{Abs}(\lambda)=[\Delta C] \varepsilon(\lambda)
$$

where $\varepsilon(\lambda)$ was the molar extinction coefficient of the sample compound at wavelength $\lambda$. When sample solution was a mixture, Eq. (13) became

$$
\Delta \mathrm{Abs}_{\text {mix }}(\lambda)=[\Delta \mathrm{BA}] \varepsilon_{\mathrm{BA}}(\lambda)+[\Delta \mathrm{BSA}] \varepsilon_{\mathrm{BSA}}(\lambda)
$$

where $[\Delta \mathrm{BA}],[\Delta \mathrm{BSA}], \varepsilon_{\mathrm{BA}}(\lambda)$ and $\varepsilon_{\mathrm{BSA}}(\lambda)$ were alternation of concentration of benzoic acid and benzenesulfonic acid, molar extinction coefficient at wavelength $\lambda$ of benzoic acid and benzenesulfonic acid, respectively.

The calculated values of the concentration variations of benzoic acid and benzenesulfonic acid were $2.0 \mu \mathrm{M}$ and $4.6 \mu \mathrm{M}$ in cases of A and B in Fig. 3, respectively. In other words, sample concentrations after passing through the mini-bed were increased from $0.100 \mathrm{mM}$ to $0.1020 \mathrm{mM}$ for benzoic acid and from $0.100 \mathrm{mM}$ to $0.1046 \mathrm{mM}$ for benzenesulfonic acid by applications of voltage on the mini-bed. Therefore it was suggested that benzenesulfonic acid was released more easily from the packing supports than benzoic acid by application of voltage. Therefore it seemed that the state of the molecular interaction between benzenesulfonic acid molecule and the surface of packing supports was weaker than the interaction between benzoic acid molecule and supports.

The degree of the interaction between solute and stationary phase is generally estimated by the capacity factor of solute in chromatography. For this purpose, liquid chromatography was used for the estimation of

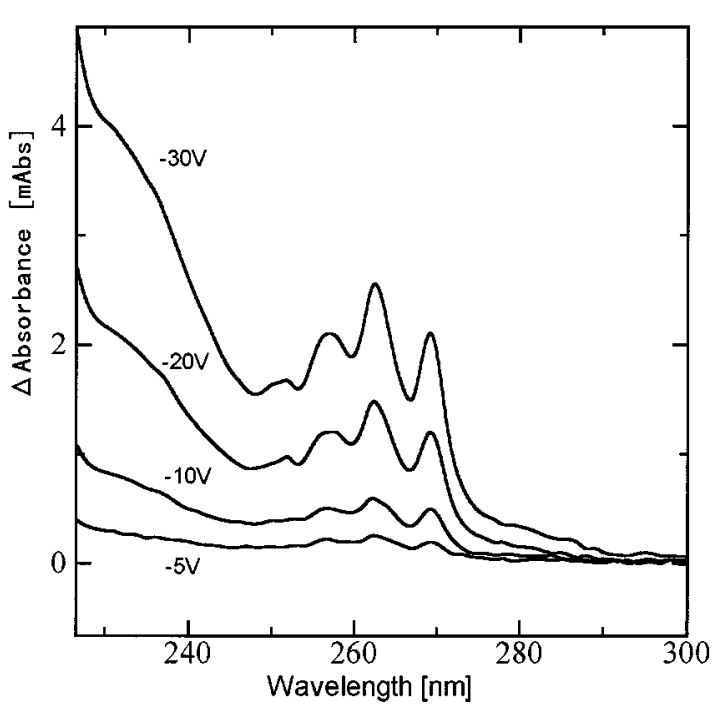

Fig. 4 Spectra at peak maximum obtained at different applied pulse voltages: $-30 \mathrm{~V},-20 \mathrm{~V},-10 \mathrm{~V},-5 \mathrm{~V}$ were applied for 6 s. Sample solution: aqueous solution containing the mixture of $0.01 \mathrm{mM}$ benzoic acid potassium salt and $0.1 \mathrm{mM}$ benzenesulfonic acid sodium salt. the degree of the interactions of benzoic acid and benzenesulfonic acid. The capacity factors of benzenesulfonic acid and benzoic acid obtained were 0.23 and 0.33 , respectively. Therefore, it is suggested that the interaction of benzenesulfonic acid molecule with the surface of packing is weaker compared to that of benzoic acid.

Dependence of concentration ratio of benzoic acid in peaks on applied voltage

Pulsed voltages of $-30 \mathrm{~V},-20 \mathrm{~V},-10 \mathrm{~V}$, and $-5 \mathrm{~V}$ were applied for $6 \mathrm{~s}$ on the mini-bed with sample solution of an the mixture of $0.1 \mathrm{mM}$ benzenesulfonic acid sodium salt and $0.01 \mathrm{mM}$ benzoic acid potassium salt aqueous solution, peaks were induced. The spectra obtained at each peak maxima are shown in Fig. 4. When the applied voltage altered from $-30 \mathrm{~V}$ to $-5 \mathrm{~V}$, the decrements of absorbance at $240 \mathrm{~nm}$ were larger than at $264 \mathrm{~nm}$. By the comparison with spectra in Fig. 3 , the absorbance at $240 \mathrm{~nm}$ was supposed to belong mostly to benzoic acid and that at $264 \mathrm{~nm}$ to benzenesulfonic acid. Therefore the decrement ratios of benzoic acid and benzenesulfonic acid might not be equal.

The values for concentration increments ratio, $\Delta C / C_{0}$, of benzoic acid and benzenesulfonic acid were estimated from Fig. 4 by using Eq. (14). Estimated values are plotted in Fig. 5. The molar percentages of benzoic acid in the sum of the amounts of the two released compounds were also plotted. It was clear that benzenesulfonic acid was more easily released than benzoic acid with application of the same voltage.

The mole percentage of benzoic acid within the sum of the amounts of the two released compounds depended on the strength of applied electric field. With the

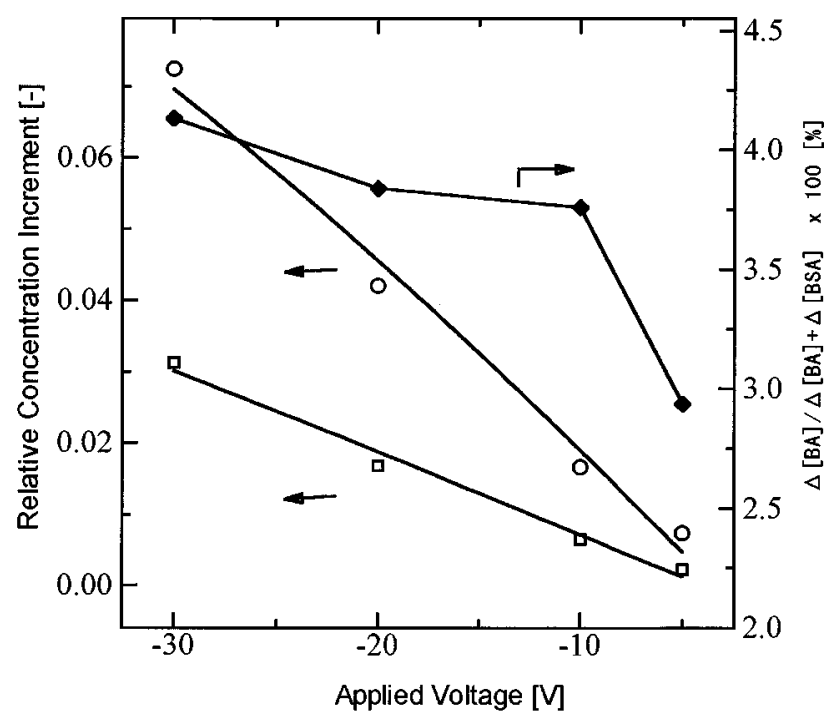

Fig. 5 Relative concentration variations induced by applied electric field. Sample: aqueous solution containing benzoic $\operatorname{acid}(\square)$ and benzenesulfonic $\operatorname{acid}(\odot)$. Closed square: mole percentage of benzoic acid. Other conditions are the same as Fig. 4. 
application of $-30 \mathrm{~V}$, the molar percentage of benzoic acid in total released compounds is $4.1 \%$, however it decreased to $2.9 \%$ with application of $-5 \mathrm{~V}$. The alternation of mole percentage will be reflected in two samples' distributions around the surface of packing supports.

Increments of concentrations of benzenesulfonic acid and benzoic acid in Fig. 5 were expressed by the following equations by using curve fitting.

$$
\Delta \mathrm{BSA} / \mathrm{BSA}=1.24 /(0.26 \exp (0.015 V)+1)-1
$$

$$
\Delta \mathrm{BA} / \mathrm{BA}=1.31 /(0.31 \exp (0.005 V)+1)-1
$$

where BSA and BA were benzenesulfonic acid and benzoic acid, respectively. These expressions are based on Eq.(12), therefore estimated capacity factors without applied voltage for benzenesulfonic acids and benzoic acid obtained from Fig. 5 were 0.26 and 0.31 , respectively. These values were nearly equal to the values obtained by experiment using a chromatographic system. Namely, the estimated values of 0.26 and 0.31 correspond to the experimental values of 0.23 and 0.33 , respectively.

The estimated values of $-z F f / R T$ for benzoic acid and benzenesulfonic acid are 0.005 and 0.015 , respectively. They are not equal. The $-z F f / R T$ value of benzenesulfonic acid was three times larger than that of benzoic acid. However, by the assumption, the proportional factor $f$ was constant. It might be reasonable to think that these phenomena induced by applied voltage were affected by the alternation around the surface of packing materials. This difference will come from the nonuniformity of the solute distribution around the packing surface. Therefore the value of $f$ would depend on the kind of chemical compound, concentrations, and/or the matrix of sample solution.

The exact mechanism of sample release induced by application of voltage was not fully understood. The phenomena were fairly reproducible. It is very interesting that those phenomena recognize the direction of electric field. The action of the releasing sample solute from the surface of packing supports was equal to alternation of capacity factors due to the application of voltage. By using this alternation, it will be possible that the gradient mode of stationary phase could be achieved by the application of electric field along the column.

\section{References}

1. V. Pretorius, B. J. Hokins and J. D. Schieke, J. Chromatogr., 99, 23 (1974).

2. T. Tsuda (ed.), "Electric Field Applications in Chromatography, Industrial and Chemical Process", VCH, Weinheim, 1995.

3. T. Tsuda, Anal. Chem., 60, 1677 (1988).

4. S. Kitagawa and T. Tsuda, J. Microcol. Sep., 6, 91 (1994).

5. T. Tsuda, LC-GC Intl., 5(9), 26 (1992).

6. E. R. Verheiji, U. R. Tjaden, W. M. A. Niessen and J. van der Greef, J. Chromatogr., 554, 339 (1991).

7. S. Kitagawa, A. Tsuji, H. Watanabe, M. Nakashima and T. Tsuda J. Microcol. Sep., 9, 347 (1997).

8. S. Kitagawa, H. Watanabe, M. Nakashima and T. Tsuda, Chromatography, 16, 170 (1995).

9. H. Watanabe, K. Nakashima, S. Kitagawa and T. Tsuda, 56th Symposium on Analytical Chemistry, May 11 - 12, 1995, Osaka (abstract pp. 135 - 136)

10. S. R. Rudge, S. K. Basak and M. R. Ladisch, AIChE J., 39, 797 (1993).

11. R. C. Weast and J. G. Grassell (ed.), "Handbook of Data on Organic Compounds, 2nd Edition Volume II Be-Bi”, CRC, Boca Raton, 1989.

(Received November 26, 1997) (Accepted February 5, 1998) 